\title{
Quantum dots with Rashba spin-orbit coupling
}

\author{
M. Governale \\ Institut für Theoretische Festkörperphysik, Universität Karlsruhe, D-76128 Karlsruhe, Germany
}

(Dated: April 2, 2018)

\begin{abstract}
We present results on the effects of spin-orbit coupling on the electronic structure of few-electron interacting quantum dots. The ground-state properties as a function of the number of electrons in the $\operatorname{dot} N$ are calculated by means of spin density functional theory. We find a suppression of Hund's rule due to the competition of the Rashba effect and exchange interaction. Introducing an in-plane Zeeman field leads to a paramagnetic behavior of the dot in a closed shell configuration, and to spin texture in space.
\end{abstract}

PACS numbers: 73.21.La,71.15.Mb,75.75.+a

Spin-related phenomena have attracted great attention recently as they are the key ingredient in the emerging field of spintronics [1]. Among these, spin-orbit (SO) coupling mechanisms in semiconductors provide a basis for device applications, and a source of interesting physics, especially in systems with reduced dimensionality. Transport through chaotic quantum dots in the presence of $\mathrm{SO}$ interaction has been studied both experimentally [2] and theoretically 3 , 田, while the effect of SO coupling on the spin lifetime has been investigated in Ref. [5]. Here, we are interested in how the electronic properties of fewelectron quantum dots, such as the addition energy [6, 7] or the spin properties of the dot ground state[8], are affected by Rashba SO interaction [9, 10]. These questions are interesting from the theoretical point of view for the following reasons. First, the Rashba effect has a different form than the usual SO coupling term in real atoms. Second, the tunability of the Rashba effect 11, 12, 13] allows dramatic SO effects to occur in quantum dots with few electrons; in real atoms this requires heavy nuclei, and hence a more complicated electronic structure.

We start by describing the physics of a quasi zerodimensional system with Rashba SO at the noninteracting electron level, providing analytical results for the single-particle spectrum when the SO coupling can be treated as a perturbation. Then, we introduce the electron-electron interaction in the framework of Spin Density Functional Theory (SDFT) 14. This allows us to study how the addition spectrum is modified by varying the strength of SO coupling. Studying the spin properties of the many-particle ground state, we find a suppression of Hund's rule, when the SO coupling can still be treated as a perturbation for the single-particle problem. For higher strengths it affects the single-particle spectrum so strongly that it gives rise to a completely different addition spectrum. The introduction of an in-plane magnetic field, leads to a paramagnetic behavior of the dot in a closed shell configuration, and to spin texture in space.

Quantum dots are often realized by lateral confinement of a two dimensional electron gas (2DEG) obtained in a heterostructure. Due to the lack of inversion symmetry along the growth direction $z$ of the heterostructure 9,10 , the electrons in the 2DEG are subject to the Rashba spinorbit coupling Hamiltonian

$$
H_{\mathrm{so}}=\frac{\hbar k_{\mathrm{so}}}{m}\left(\sigma_{x} p_{y}-\sigma_{y} p_{x}\right) .
$$

The strength of the SO coupling, here denoted as $k_{\mathrm{so}}$, can be tuned by changing the asymmetry of the quantum well via externally applied voltages, as shown in several experimental studies 11, 12, 13.

It is interesting to study the effect of the SO coupling term Eq. (1) on the quantum mechanics of a quasi zerodimensional system[15]. To this end, we consider a twodimensional quantum dot defined by a parabolic confining potential

$$
V_{\text {conf }}(x, y)=\frac{m}{2} \omega^{2}\left(x^{2}+y^{2}\right) .
$$

Thus, the single-particle Hamiltonian in the effectivemass approximation reads

$$
H=\frac{p_{x}^{2}+p_{y}^{2}}{2 m}+V_{\text {conf }}(x, y)+H_{\text {so }} .
$$

In the absence of SO coupling the eigen-energies are

$$
E_{M}^{(0)}=\hbar \omega(M+1)
$$

with $M$ being a non-negative integer. A degenerate subspace $\mathcal{S}_{M}$ of dimension $D_{M}=2(M+1)$, where the factor 2 is due to spin, is associated to each energy $E_{M}^{(0)}$.

We will now treat $H_{\text {so }}$ as a perturbation. This is valid as long as $k_{\mathrm{so}} l_{\omega} \ll 1$, where $l_{\omega}$ is the oscillator length $\sqrt{\hbar /(m \omega)}$. We obtain for the second-order eigen-energies

$$
\tilde{E}_{M, i, \sigma}=E_{M}^{(0)}+\hbar \omega\left(k_{\mathrm{so}} l_{\omega}\right)^{2}[2(i-1)-(M+1)],
$$

where $i=1, \cdots, M+1$, and $\sigma= \pm 1$ is the quantum number relative to $\sigma_{z}$, i.e. the spin projection along $z$. As the single-particle levels will play an important role in the following, we show an example of the low-energy part of the spectrum calculated using Eq. (5) together with the results of numerical diagonalization in the upper panel 
of Fig. 1. From the perturbative treatment the following conclusions can be drawn: 1) Each degenerate level $E_{M}^{(0)}$ is split in $M$ sublevels, each of which is double degenerate due to Kramers theorem; 2) Spin rotational invariance is broken but still the eigenstates are (to this order in perturbation theory) eigenstates of $\sigma_{z}$. From conclusion 1 ) we can infer that SO coupling changes the addition spectrum of the dot, while conclusion 2) tells us that the Rashba effect will not influence the lifetime of the eigenstates of $\sigma_{z}$. For values of $k_{\mathrm{so}}$ for which perturbation theory breaks down, the eigen-energies are of course still grouped in Kramers-degenerate sublevels [as shown in the inset of Fig. [1(a)], although it can happen that different sublevels have almost the same energy.

To introduce the Coulomb interaction between the electrons we use spin density functional theory (SDFT), in the local density approximation 14. We write the Kohn-Sham equation 16$]$ in spinor notation

$$
\begin{aligned}
& {\left[\left(-\frac{\hbar^{2}}{2 m} \nabla^{2}+V_{\text {conf }}+V_{\text {coul }}\right) \mathbf{1}+H_{\text {so }}+\mathbf{V}_{\text {exch-corr }}\right] \Phi_{j}} \\
& =\varepsilon_{j} \Phi_{j},
\end{aligned}
$$

where $V_{\text {coul }}$ is the Hartree potential, $\mathbf{V}_{\text {exch-corr }}$ the exchange-correlation potential, $\mathbf{1}$ the identity matrix in spin space, and $\Phi_{j}$ is a two-components spinor. The spindependent exchange-correlation potential is introduced following Ref. [14]. It is in general non diagonal in spin space (it becomes diagonal in the basis that diagonalizes the spin-density matrix). For the exchange-correlation energy we use the Tanatar and Ceperly parameterized form [17]; in particular for the case of partial spin polarization we use the interpolation scheme of Refs. [8, 14. We solve Eq. (6) self-consistently by discretizing it in real space. The addition energy (also called capacitive energy by some authors (7) is defined as $E_{\text {add }}=\mu(N+1)-\mu(N)$, where $\mu(N)$ is the chemical potential for the dot with $N$ electrons, i.e. the energy needed to add the $N$-th electron to the system containing already $N-1$ electrons. We compute the chemical potential by means of Slater's rule 18, in order to minimize numerical errors due to double differentiation.

Now, we focus on realistic dots obtained in an InAs heterostructure, where the Rashba effect can be quite large 13. We use for the electron effective mass the value $m=0.022 m_{0}$, with $m_{0}$ being the free-electron mass; and for the dielectric constant $\epsilon=14.6 \epsilon_{0}$, being $\epsilon_{0}$ the one of vacuum.

In a quantum dot without SO interaction we expect peaks in the addition energy when the number of electrons $N$ equals a magic numbers, i.e at those integer values which correspond to a closed shell configuration. For a parabolic dot the first magic numbers are 2,6,12, see Eq. (4) and the discussion below it. Besides these peaks for $N$ coinciding with a magic number, some additional peaks are expected for a number of electrons correspond- a)
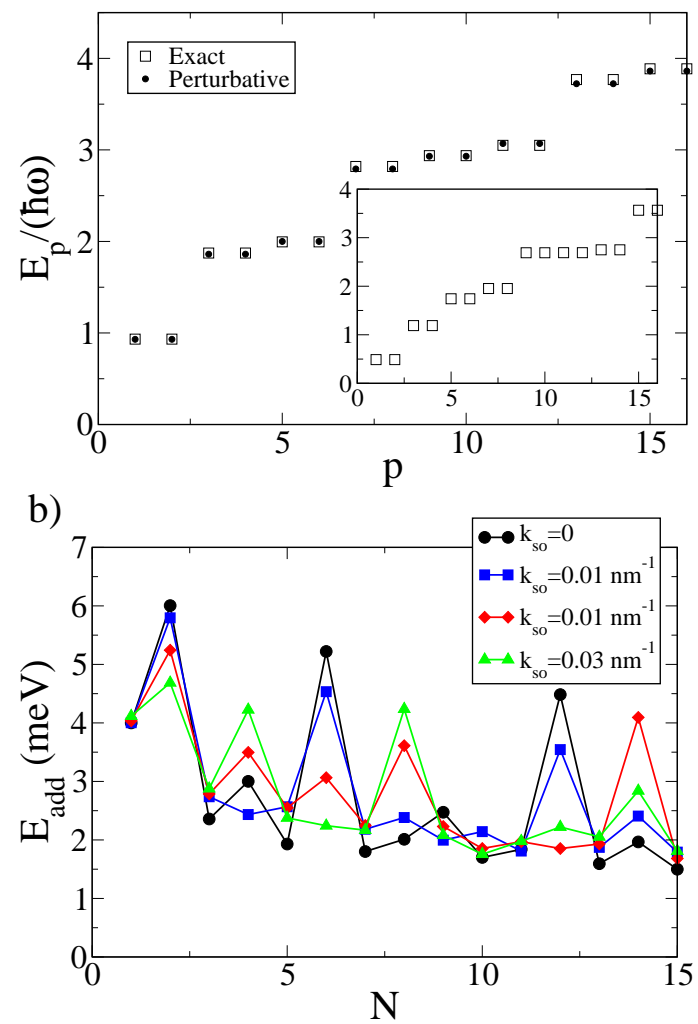

FIG. 1: (a) Low-energy part of the single-particle spectrum for the dot, calculated both by perturbation theory (dots) and by numerical diagonalization (squares) for $k_{\mathrm{so}} l_{\omega}=0.2633$ [this value corresponds to the data for $k_{\mathrm{so}}=0.01 \mathrm{~nm}^{-1}$ for the addition energies of the realistic dot shown in panel (b)]. The label $p$ is an index that enumerates the eigenstates in order of ascending energy. Inset: Low-energy part of the singleparticle spectrum calculated numerically for $k_{\mathrm{so}} l_{\omega}=0.7896$ [this value correspond to $k_{\text {so }}=0.03 \mathrm{~nm}^{-1}$ in panel (b)] In this case the SO coupling dominates the single-particle spectrum. (b) Addition energy vs number of electrons in the dot for different values of the SO coupling strength. In this figure and in the following ones the dot is defined by a confining potential of strength $\hbar \omega=5 \mathrm{meV}$.

ing to a half-filled shell due to Hund's rule. In this situation the electrons in the half-filled shell have parallel spins to gain exchange energy. For the parabolic dot under consideration the first Hund's-rule peaks are located at $N=4$, and $N=9$. Both these kind of peaks can be seen in Fig. 1 (b), for the case when no SO coupling is present (filled dots). Switching on the SO interaction leads to a change in the single-electron levels of the dot, and such a change is reflected in the addition energy. This effect can be seen in Fig. 1 (b), where the addition energy is plotted for several values of the SO coupling strength, ranging from a situation where the perturbative treatment Eq. (5) is still valid, to one where the SO coupling dominates the single-particle spectrum. Due to the presence of Kramers-degenerate sublevels in the free-electron 
spectrum, peaks tend to be present for even number of electrons in the dot. The fact that a degenerate level $E_{M}^{(0)}$ is split in $M$ Kramers-degenerate sublevels (in the perturbative regime), leads to a suppression of the Hund's rule: in a half-filled level to maximize the total spin the electrons should be allocated one per sublevel, but this has an energy cost equal to the sublevel splitting; if the sublevel splitting is larger than the gain in exchange energy, then the spin polarization for a half-filled level is suppressed. This is indeed what we see by analyzing the spin properties of the SDFT ground-state wave-function. At this point it is important to stress that due to the tunability of the SO coupling strength 11, 12, 13], it is possible to investigate experimentally the effect of the Rashba term on the addition spectrum of few-electrons quantum dots (addition spectra were measured by Tarucha et al. [6] ), and the transition from weak $\left(k_{\mathrm{so}} l_{\omega} \ll 1\right)$ to strong SO coupling.

We now investigate the effect of an in-plane magnetic field. Due to the fact that the system is invariant under rotation around the $z$-axis, we can choose the direction of the in plane magnetic field arbitrarily without losing any generality. We introduce a magnetic field $B$ along $x$, which does not affect directly the orbital motion, but couples to the $x$-component of the total spin, giving rise to a Zeeman term, $H_{z}=\hbar \omega_{z} S_{x} / 2$, where $S_{x}=\sum_{i=1, N} \sigma_{x}^{(i)}$, and $\hbar \omega_{z}=\mu_{\mathrm{B}} g^{*} B$, with $\mu_{\mathrm{B}}$ being Bohr's magneton, and $g^{*}$ the g-factor. We consider now a dot in a closed-shell configuration, namely we take $N=2$ and $N=6$. In the case of vanishing SO coupling and in the independentelectron picture, such a system does not respond to the in-plane magnetic field for Zeeman splitting smaller than the level splitting $\left(\omega_{z}<\omega\right)$. The situation changes when the Rashba term is introduced; the ground state of the dot exhibits now some net-spin polarization. In the upper panel of Fig. 2, the average value of $S_{x}$ is plotted vs magnetic field, showing how the system gets magnetized even in a closed-shell configuration due to the interplay of SO coupling and Zeeman splitting. The average values of $S_{y}$ and $S_{z}$ remain equal to zero. In the lower panel of Fig. 2, the variation of the ground state energy with magnetic field is plotted vs magnetic field. It shows a decrease with increasing field (which is well fitted by a parabola), yielding a positive susceptibility $\chi=-\partial^{2} E / \partial B^{2}$. Thus, we can conclude that the dot in a closed shell configuration exhibits a paramagnetic behavior. This is in contrast to what happens in real atoms, where a closed shell gives a diamagnetic response due to orbital degrees of freedom (Larmor diamagnetism) [19], while in our case the Larmor term is suppressed by the two-dimensionality of the dot. This paramagnetic behavior 20 is due to the singleparticle eigen-states (see below), but it persists when the electron-electron interaction is present (it is enhanced by it), as shown in Fig. 2.

In the limit of $k_{\mathrm{so}} l_{\omega} \omega / \omega_{z} \ll 1$, and $w_{z}<w$, it is pos-
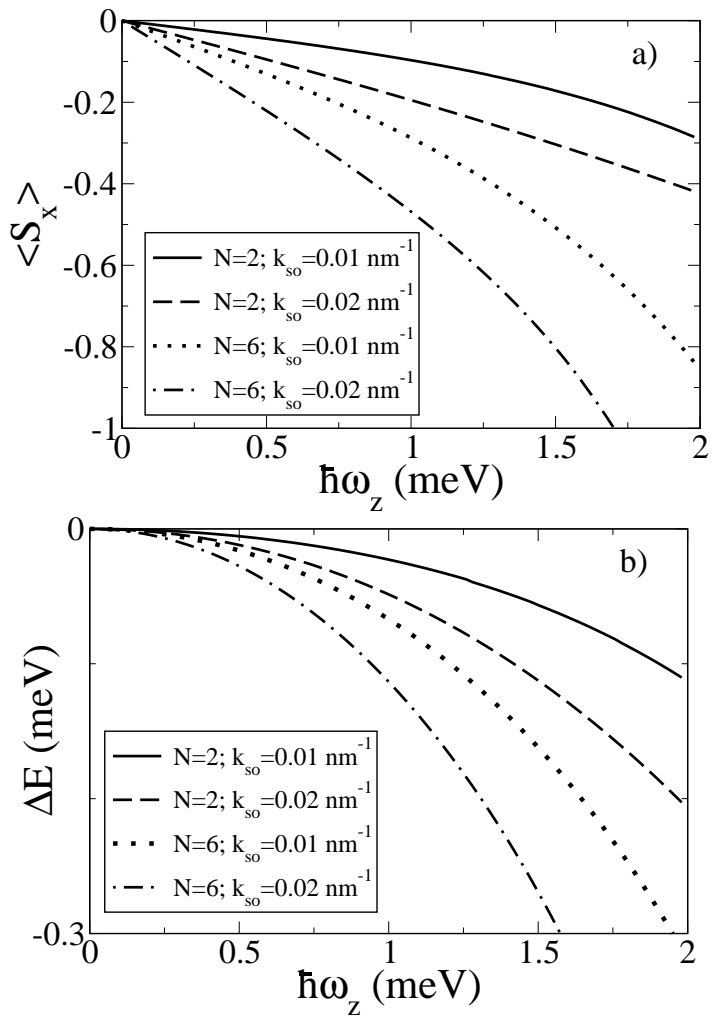

FIG. 2: (a) Average values of $S_{x}$ vs the strength of the in-plane magnetic field, computed by means of SDFT, for two closed shell configurations $(N=2$ and $N=6)$ and for different strength of the spin-orbit interaction. Without the Rashba term, $\left\langle S_{x}\right\rangle$ would be zero.

(b) Variation of the ground state energy $\Delta E=E(B)-E(0)$ vs the strength of the in-plane magnetic field, computed by means of SDFT. The ground state energy shows a quadratic dependence on the in-plane magnetic field: $E(B)=E(0)-$ $A B^{2}$, with $A>0$. The susceptibility is positive and the system is paramagnetic.

sible to obtain a perturbative expression in $H_{\text {so }}$ for the single-particle eigen-energies:

$$
\begin{aligned}
\tilde{E}_{M, i, \sigma}= & E_{M}^{(0)}+\sigma \frac{\hbar \omega_{z}}{2}-\frac{\hbar \omega}{2}\left(k_{\mathrm{so}} l_{\omega}\right)^{2} \\
& \left\{1+\frac{\omega^{2}}{\omega^{2}-\omega_{z}^{2}}\left[1+\sigma(2 i-1) \frac{\omega_{z}}{\omega}\right]\right\},
\end{aligned}
$$

where $i=1 \cdots M+1, \sigma= \pm 1$ is the quantum number relative to the projection of spin in the direction of the magnetic field, i.e $\sigma_{x}$, and $E_{M}^{(0)}$ are the energies given in

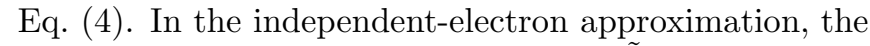
energy of a closed shell $E_{\mathrm{cs}}(M)=\sum_{i, \sigma} \tilde{E}_{M, i, \sigma}$ reads

$$
\begin{aligned}
E_{\mathrm{cs}}(M)= & 2(M+1) E_{M}^{(0)} \\
& -(M+1) \hbar \omega\left(k_{\mathrm{so}} l_{\omega}\right)^{2}\left[1+\frac{\omega^{2}}{\omega^{2}-\omega_{z}^{2}}\right] .
\end{aligned}
$$

Expanding Eq. (8) in $\omega_{z} / \omega$ we get for the magneticfield dependent part of the closed-shell energy $-(M+$ 

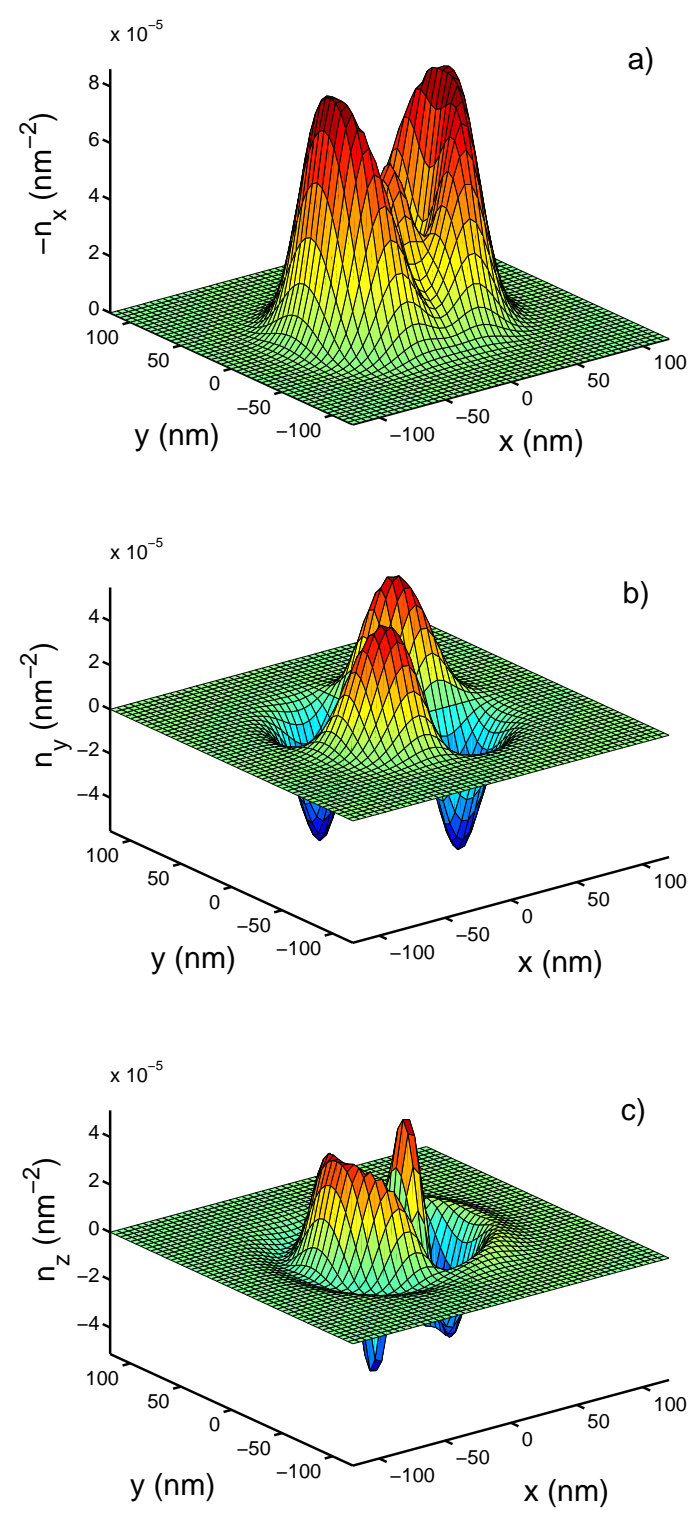

FIG. 3: Spin density projected along $x$ (a), $y(\mathrm{~b})$, and $z$ (c), computed by means of SDFT, for a dot with $N=6$ electrons, $k_{\mathrm{so}}=0.02 \mathrm{~nm}^{-1}$, and $\hbar \omega_{z}=1 \mathrm{meV}$. In (a), for ease of visualization, we plot $-n_{x}$ instead of $n_{x}$. Spatial integration of $n_{y}$ and $n_{z}$ gives zero, yielding a zero average for the corresponding total-spin components.

1) $\hbar \omega\left(k_{\mathrm{so}} l_{\omega}\right)^{2}\left(\omega_{z} / \omega\right)^{2}+\mathcal{O}\left[\left(\omega_{z} / \omega\right)^{4}\right]$, which explains the parabolic behavior seen in Fig. 2. From Eq. (8) we get a paramagnetic contribution to the susceptibility due to a closed-shell

$$
\frac{\chi_{M}}{\left(g^{*} \mu_{\mathrm{B}}\right)^{2}}=2(M+1) \omega^{3}\left(k_{\mathrm{so}} l_{\omega}\right)^{2} \frac{\omega^{4}-\omega_{z}^{4}}{\hbar\left(\omega^{2}-\omega_{z}^{2}\right)^{4}},
$$

which in the limit of $\omega_{z} / \omega \ll 1$ is just a positive constant: $\chi_{M} /\left(g^{*} \mu_{\mathrm{B}}\right)^{2}=2(M+1)\left(k_{\mathrm{so}} l_{\omega}\right)^{2} /(\hbar \omega)$.

It is interesting to have a closer look at the spin-density for the magnetized dot. In Fig. 3 the projections of the spin density along the $x$-, $y$-, and $z$-axis are shown for a dot containing six electrons in the presence both of SO coupling and of a Zeeman field. As it is clearly visible, the system shows spin texture in space, this is due to the fact that no common spin-quantization axis exists anymore (a similar situation occurs in quantum wires with strong spin-orbit coupling 21].)

In conclusion, we have studied the effect of Rashba spin-orbit interaction on the addition energy, and on the spin properties of a few-electron quantum dot by means of spin density functional theory. In particular, we have found a suppression of Hund's rule, for small $k_{\text {so }}$ values, for which perturbation theory in $H_{\text {so }}$ still holds. An additional in-plane magnetic field (Zeeman field) leads to a paramagnetic behavior of the dot in a closed shell configuration, and to spin texture in space.

This work was supported by the Center for Functional Nanostructures at the University of Karlsruhe. We thank J. C. Cuevas, M. Macucci, and U. Zülicke for useful discussions.

[1] S. A. Wolf et al, Science 294, 1488 (2001).

[2] J. A. Folk et al., Phys. Rev. Lett. 86, 2102 (2001)

[3] B. I. Halperin et al., Phys. Rev. Lett. 86, 2106 (2001).

[4] I. L. Aleiner, and V. I. Fal'ko, Phys. Rev. Lett. 87, 256801 (2001).

[5] A. V. Khaetskii, and Y. V. Nazarov, Phys. Rev. B 61, 12639 (2000).

[6] S. Tarucha, D. G. Austing, T. Honda, R. J van der Hage, and L. P. Kouwenhoven, Phys. Rev. Lett. 77, 3613 (1996).

[7] M. Macucci, K. Hess, and G.J. Iafrate, Phys. Rev. B 55, R4879 (1997).

[8] M. Koskinen, M. Manninen, and S. M. Reimann, Phys. Rev. Lett. 79, 1389 (1997).

[9] E. I. Rashba, Fiz. Tverd. Tela (Leningrad) 2, 1224 (1960), [Sov. Phys. Solid State 2, 1109 (1960)].

[10] G. Lommer, F. Malcher, and U. Rössler, Phys. Rev. Lett. 60, 728 (1988).

[11] J. Nitta, T. Akazaki, H. Takayanagi, and T. Enoki, Phys. Rev. Lett. 78, 1335 (1997).

[12] T. Schäpers et al., J. Appl. Phys. 83, 4324 (1998).

[13] D. Grundler, Phys. Rev. Lett. 84, 6074 (2000).

[14] U. von Barth and L. Hedin, J. Phys. C 51629 (1972).

[15] The effect of the spin-orbit interaction arising from lateral confinement in the dot on the single-particle spectrum has been discussed by O. Voskoboynikov, C. P. Lee, and O. Tretyak, Phys. Rev. b 63, 165306 (2001).

[16] W. Kohn and L. J. Sham, Phys. Rev 140, A1133 (1965).

[17] B. Tanatar and D. M. Ceperley, Phys. Rev. B 39, 5005, (1989).

[18] J. P. Perdew and A. Zunger, Phys. Rev. B 23, 5048 (1981).

[19] For a closed atomic shell Van Vleck paramagnetism is absent.

[20] The magnetic susceptibility of free electrons subject 
to Rashba spin-orbit coupling has been discussed by I. I. Boiko, E. I. Rahsba, Fiz. Tverd. Tela Sov. (Leningrad)2 1874 (1960), [Phys. Solid State 2, 1692 (1960)]; Y. A. Bychkov and E. I. Rashba, J. Phys. C
17, 6039, (1984).

[21] M. Governale, and U. Zülicke, cond-mat/0201164 (2002). 